\title{
Do laws impact opioids consumption? A breakpoint analysis based on Italian sales data
}

\section{Umberto Maria Musazzi' \\ Paolo Rocco' \\ Cinzia Brunelli ${ }^{2,3}$ \\ Luisa Bisaglia ${ }^{4}$ \\ Augusto Caraceni ${ }^{2,3}$ \\ Paola Minghetti'}

'Department of Pharmaceutical Sciences, University of Milan, Milan, Italy; ${ }^{2}$ Palliative Care, Pain Therapy and Rehabilitation Unit, Fondazione IRCCS Istituto Nazionale Dei Tumori, Milano, Italy; ${ }^{3}$ European Palliative Care Research Centre (PRC), Department of Cancer Research and Molecular Medicine, Faculty of Medicine, Norwegian University of Science and Technology (NTNU), Trondheim, Norway; ${ }^{4}$ Department of Statistical Sciences, University of Padua, Padua, Italy

This article was published in the following Dove Press journal: Journal of Pain Research

\begin{abstract}
Purpose: In Italy, where the adoption of opioid analgesics in pain management has been historically poor, an increase in opioids consumption occurred between 2000 and 2015. The aim of this study is to assess, through specific time series analyses for trend changes, the impact of different intervening factors - such as the availability of new drugs, the observance of clinical guidelines, changes in prescription regulations, and in reimbursement policies - on opioids sales to community pharmacies in Italy, focusing on the time period 2000-2010.
\end{abstract}

Materials and methods: Five opioids were considered: codeine, tramadol, buprenorphine, morphine, and fentanyl. The analysis is based on sales data collected at wholesale distributors. For each one of the five drugs, time series of the number of Defined Daily Doses per thousand inhabitants per day in the period 2000-2010 were analyzed, and an estimation of breakpoints was performed using segmented linear regression.

Results: Drug sales underwent a sharp increase in 2000-2010, although on different scales. Segmented regression analysis highlighted different potential breakpoints, corresponding to either a significant change in value and/or in slope. Sales of the five opioids were affected by at least one relevant event, often due to a synergy of regulatory, marketing, and technological factors. The effect of reimbursement changes has proved important.

Conclusion: Between 2000 and 2010, regulatory, technological, and reimbursement changes significantly influenced opioid sales to community pharmacies in Italy. The sales of relatively new drug products seem to be less influenced by changes in reimbursement and regulatory policies than that of more established products, suggesting that physicians are more comfortable with "old" drugs, since their clinical use is supported by established clinical guidelines and protocols. Keywords: opioids sales, community pharmacy, reimbursement policy, legislation changes, breakpoint estimation

\section{Introduction}

In the last decades, the management of pain caused by chronic and progressive incurable illnesses has become a central focus of healthcare systems in assuring an acceptable quality of life to patients. ${ }^{1}$ Although the World Health Organization (WHO) has introduced specific guidance for pain management, ${ }^{2}$ the adoption of opioids analgesics in pain management has remained poor in some western countries. ${ }^{3}$ Some authors and the WHO ascribed the under-treatment of pain with opioids to stringent legislation and control, ${ }^{3,4}$ while other authors showed that factors such as the marketing of new drugs, changes in reimbursement policies, cultural changes, and increased patients' awareness also had a significant impact. ${ }^{5-7}$
Correspondence: Paola Minghetti Department of Pharmaceutical Sciences, Università Degli Studidi Milano, Via G.

Colombo, 7I - 20133 Milan, Italy

Tel +39250324639

Fax +39250324657

Email paola.minghetti@unimi.it 
On the other hand, the abuse potential of opioids cannot be underestimated. In some countries, an excessive increase in opioid prescription in the last 15 years has led to an opioid epidemic, which, although relatively absent in Europe, has gained the proportion of a public health crisis in the United States. Therefore, efforts are directed toward gaining a deeper understanding of pain and toward the development of novel, nonaddictive analgesics. ${ }^{8,9}$

In a previous report, opioids sales from 2000 to 2010 were analyzed separately by distribution setting, to highlight the differences between community pharmacies and hospitals. ${ }^{10}$ The study showed an overall increase in opioids sales, with a much higher relative growth in community pharmacies. However, the reasons for that increase were not rigorously analyzed.

The aim of this study is to assess, through specific time series analyses for trend changes, the impact of legislation, reimbursement by the Italian National Health System (NHS), and availability of new drugs or new dosage forms on the sales of opioids for pain management to Italian community pharmacies between 2000 and 2010. Italy is an ideal setting for such an analysis, since a clear-cut steady increase in opioid consumption occurred in that period. ${ }^{10}$ After 2011 , the increase seemed to level off. ${ }^{11}$ The statistical analysis focused on the period 2000-2010, when the most important reimbursement and legislative changes took place.

\section{Materials and methods}

Original data were quarterly number of packs sold to community pharmacies in the period 2000-2010 (supplied by IMS Health S.r.l, Italy). Only five opioids used in pain management were considered: codeine, tramadol, buprenor- phine, morphine, and fentanyl (Table 1). The data obtained from sales of oxycodone and hydromorphone - introduced in Italy, respectively, in 2005 and 2008 - were not analyzed due to the limited sample size in the considered time series. Methadone was also excluded from the analysis, since it is mainly prescribed to patients with opioid addiction. For each specific commercial product, the total amount in milligrams was calculated by multiplying the number of packs sold by the number of units per pack by the amount of drug (mg) per unit.

The dosage forms included in the analysis were: conventional oral dosage forms for codeine and tramadol (drug products in association with paracetamol), conventional and modified release oral dosage forms, and solution for injection for tramadol and morphine. Immediate release oral dosage forms and transdermal patches for fentanyl and buprenorphine; transmucosal oral dosage forms for fentanyl and solution for injection for buprenorphine. Further details are listed in the previous report. ${ }^{10}$ Review and approval was not required for this research by an institutional review board or ethics committee, because it does not involve human subjects and only involves the use of de-identified aggregated data.

\section{Relevant events}

In this section, the most relevant events that may influence opioids sales are highlighted. In particular, changes in the regulatory framework of drug prescription for pain management or in reimbursement policies and the availability of new active substances or dosage forms (Table 1). Noteworthy, some of the events are very closely spaced, and attributing a change in sales to one unique cause proved difficult in some cases.

Table I Different steps in the Italian opioids market and prescription regulation

\begin{tabular}{lll}
\hline Line & Date & Description \\
\hline RI & first Q 200I & $\begin{array}{l}\text { Easier prescription for all opioids for patients suffering from severe } \\
\text { pain due to neoplastic or degenerative disease } \\
\text { Introduction of morphine oral solution }\end{array}$ \\
M/TI & second Q 200I & $\begin{array}{l}\text { Introduction of buprenorphine transdermal patch } \\
\text { Introduction of oxycodone }\end{array}$ \\
M/T2 & second Q 2003 & Reimbursement by the It-NHS of most opioids \\
Reiml & fourth Q 2004 & Introduction of oral transmucosal fentanyl \\
M/T4 & first Q 2005 & Introduction of generic of transdermal fentanyl \\
M/T5 & first Q 2005 & Easier prescription for tramadol \\
Rla & fourth Q 2005 & Easier prescription for opioids extended to all patients \\
R2 & third Q 2006 & Switch from reservoir to matrix technology for transdermal fentanyl \\
M/T6 & second Q 2007 & Introduction of hydromorphone \\
M/T7 & second Q2007 & Further simplification for low dose oxycodone prescription \\
R3 & third Q 2007 & Revocation of the special prescription pad for all opioids (except for \\
R4 & first Q 2008 & parenteral morphine and oral buprenorphine) \\
\hline
\end{tabular}

Note: $\mathrm{i}=1,2$, etc.

Abbreviations: R(i), i-th regulatory break; M/T(i), i-th market/technology break; Reim(i), i-th reimbursement break (a special case of regulatory break); Q, quarter. 


\section{Relevant regulatory interventions on opioids prescribing}

Changes in the regulatory framework (marked with $\mathrm{R}$ ) are summarized in Table 1 and briefly described in this paragraph.

Law n. 12 of February 8, 2001. In effect: March 6, 2001 (R1). The law introduces a simplified prescription pad (called RMR) for a number of active substances (included in Annex III bis), destined to patients suffering from severe pain due to a neoplastic or degenerative disease. The RMR prescription pad is in effect since June 12, 2001 (Decree of May 24, 2001). The original Annex III bis of 2001 contained 10 drugs: buprenorphine, codeine, dihydrocodeine, fentanyl, hydrocodone, hydromorphone, methadone, morphine, oxycodone, oxymorphone.

Decree of the Ministry of Health of June 19, 2006. In effect: July 11, 2006. Only relevant to tramadol (R1a). Tramadol (not included in Annex III bis, and prescribable with a standard non-repeat prescription, so called RNR), is excluded from the list of drugs of abuse. No changes to prescription regulations for tramadol are made.

Decree of the Ministry of Health of April 18, 2007. In effect: April 29, 2007 (R2). The active substances listed in Annex III bis can be prescribed to all patients, not only patients suffering from severe pain due to neoplastic or degenerative disease.

Decree of December 21, 2007. In effect: February 13, 2008. Only relevant to Oxycodone (R3). The prescription requirements for oral drug products containing low dosages of oxycodone are simplified. In particular, doses lower than $10 \mathrm{mg}$ can be prescribed using a standard RNR, instead of the RMR prescription needed for the other Annex III bis drugs.

Ministerial decree (Ordinanza) of June 16, 2009. In effect: June 20, 2009 (R4). Except for parenteral morphine, the medicinal products containing active substances listed in Annex III bis can be prescribed using the standard RNR instead of RMR prescription. Prescription of parenteral morphine is addressed in the subsequent Law n. 38 of March 15, 2010.

Law n. 38 of March 15, 2010. In effect: April 3, 2010. Only relevant to parenteral morphine. All the medicinal products used in pain management and reimbursed by the National Health System can be prescribed using standard RNR.

\section{Changes in reimbursement policies}

Through the years, reimbursement policies for opioid analgesics distributed through community pharmacies have changed. For some medicinal products, reimbursement by the NHS started before the period considered in the analysis. For medicinal products containing tramadol, codeine, buprenorphine, and oxycodone, on the other hand, reimbursement started after January 1, 2005. ${ }^{12}$ Initially, reimbursement for tramadol and codeine was restricted to the treatment of patients suffering from pain due to a neoplastic or degenerative disease. Such restrictions were eliminated in 2005 for codeine ${ }^{13}$ and October 2009 for tramadol. ${ }^{14}$ The only relevant change in reimbursement policy is marked with Reim1 in Table 1.

\section{Introduction of new active substances or dosage forms on the market}

The availability of new drugs or new dosage forms is marked with $\mathrm{M} / \mathrm{T}$ in Table 1 . Since the Marketing Authorization does not generally coincide with the availability on the market of a new medicinal product, the timeline in Table 1 refers to the emergence of sales figures in the analyzed data.

\section{Statistical analyses}

Opioid amounts in milligrams are standardized using the Defined Daily Dose (DDD) methodology recommended by the World Health Organization (WHO) for drug consumption studies. The official DDD values are established by the WHO Collaborating Center for Drug Statistics Methodology: transdermal and parenteral buprenorphine, $1.2 \mathrm{mg}$; oral codeine, $90 \mathrm{mg}$; sublingual/buccal fentanyl, $0.6 \mathrm{mg}$; transdermal fentanyl, $1.2 \mathrm{mg}$; parenteral morphine, $30 \mathrm{mg}$; oral morphine, $100 \mathrm{mg}$; oral tramadol, $300 \mathrm{mg} .^{15}$

For each one of the five drugs considered, the time series of the number of DDDs per thousand inhabitants per day were analyzed. Data on resident population for each year in the period 2000-2010 was obtained from official reports of the Italian National Institute of Statistics. ${ }^{16}$

After a graphical descriptive examination of the wholesales time series for the five opioids considered, the potential impact of the interventions in the regulatory and technological field was tested through the estimation of breakpoints, ie, points in which data show deviations from stability in the background trend.

For each one of the five opioids considered, the estimation of breakpoints was performed using segmented linear regression, in which a maximum four a priori unknown breakpoints are allowed. ${ }^{17}$ With four breakpoints, the considered interval of time is divided into a maximum of five segments in which the regression coefficients are kept constant, and a different model is estimated in each of these intervals. The model 
can be written through the following regression equation: $\mathrm{y}_{\mathrm{t}}=\alpha_{\mathrm{j}}+\beta_{\mathrm{j}}{ }^{*} \mathrm{t}+\varepsilon_{\mathrm{t}}$, where $\mathrm{j}=(1, \ldots, 5), \mathrm{t}=\mathrm{T}_{\mathrm{j}-1+1}, \ldots, \mathrm{T}_{\mathrm{j}}, \varepsilon_{\mathrm{t}} \sim \mathrm{N}\left(0, \sigma^{2}\right)$.

Breakpoints estimates are obtained by minimizing the residual sum of squares of the regression equation; the $95 \%$ CI for each breakpoint estimated is also reported.

Data analyses are performed using Stata 13.0 (StataCorp LLC, College Station, TX, 2013) ${ }^{18}$ and R software (R Core Team, Vienna, Austria, 2013), ${ }^{19}$ in particular the library Strucchange. ${ }^{20}$

\section{Results}

Sales data for the five selected opioids are shown in Figure 1. Both Step II and Step III drugs sales have undergone a sharp increase, although on different scales. The greatest increase is observed for fentanyl, whereas morphine shows the only negative trend.

Segmented regression analysis highlighted different potential breakpoints, corresponding to either a significant change in value and/or in slope. The segmented regression lines and the estimated breakpoints are shown in Figure 2. An overview of all the potential breakpoints is shown in Figure 3.

\section{Codeine}

Three breakpoints are estimated for the time series of codeine (Figure 2). The second breakpoint is at Reim1 (Table 1). The observed decrease in value and increase in slope may be explained with the availability of oxycodone in the fourth Quarter of 2004 and the change in reimbursement policies

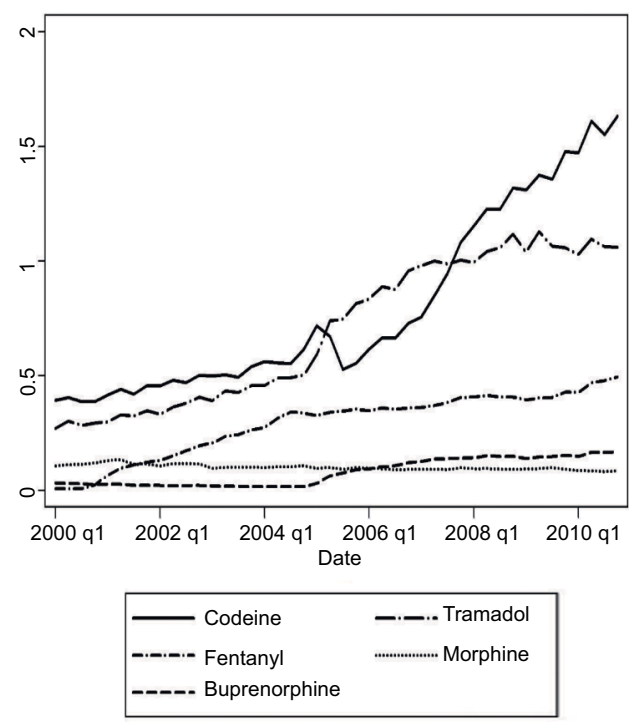

Figure I Sales of WHO Step II and Step III opioids to community pharmacies between 2000 and 2010. Data in DDDs/day/l000 inhabitants.

Abbreviations: WHO, World Health Organization; DDD, defined daily dose; q, quarter. in the first Quarter of 2005. The last breakpoint in the third Quarter of 2007 may be related to the easier prescription being extended to all patients.

\section{Tramadol}

A sharp increase (in value and slope) in the first Quarter of 2005 seems to be related to the change in reimbursement policies. Two breakpoints are located after the first Quarter of 2007, where the slope decreases (R2 and R4). Both results may be due to the fact that tramadol did not benefit from those normative interventions, despite the easier prescription (R1a) starting in the third Quarter of 2006 (Figure 2).

\section{Morphine}

Morphine shows the only decreasing trend. A first decrease in the second Quarter of 2001, despite the easier prescription (R1) and the availability of oral solution (M/T1), and a second decrease in the fourth Quarter of 2004, in conjunction with the introduction of Oxycodone and transmucosal Fentanyl. The decrease may be ascribed to a missed reimbursement policy change in the first Quarter of 2005 (Figure 2).

\section{Fentanyl}

Fentanyl shows a decrease in slope in the first Quarter of 2004, following the availability of buprenorphine transdermal patches (second Quarter 2003). The decline continues in the third Quarter of 2007, followed by an upward trend in slope, after the Decree of second Quarter 2009 (R4) (Figure 2).

\section{Buprenorphine}

Buprenorphine sales start in a downward trend that shows a first reversal upon the introduction of transdermal patches in the second Quarter of 2003. The promising results of the transdermal dosage form are hidden by the breakpoint occurring with the change in reimbursement policies in the first Quarter of 2005 (Figure 2).

\section{Discussion}

Opioids sales in community pharmacies in Italy showed a positive trend in the period 2000-2010 (Figure 1), in agreement with the trend observed in the European Union.,21 Although regulatory interventions impacted sales in both community pharmacies and hospitals, ${ }^{10}$ the sales in community pharmacies are particularly interesting for determining the role of a less stringent regulation for opioids prescription. Indeed, unlike hospitals where sales of different opioids are more affected by payer's policies and, probably, by guidelines, in community pharmacies, sales are more directly related 

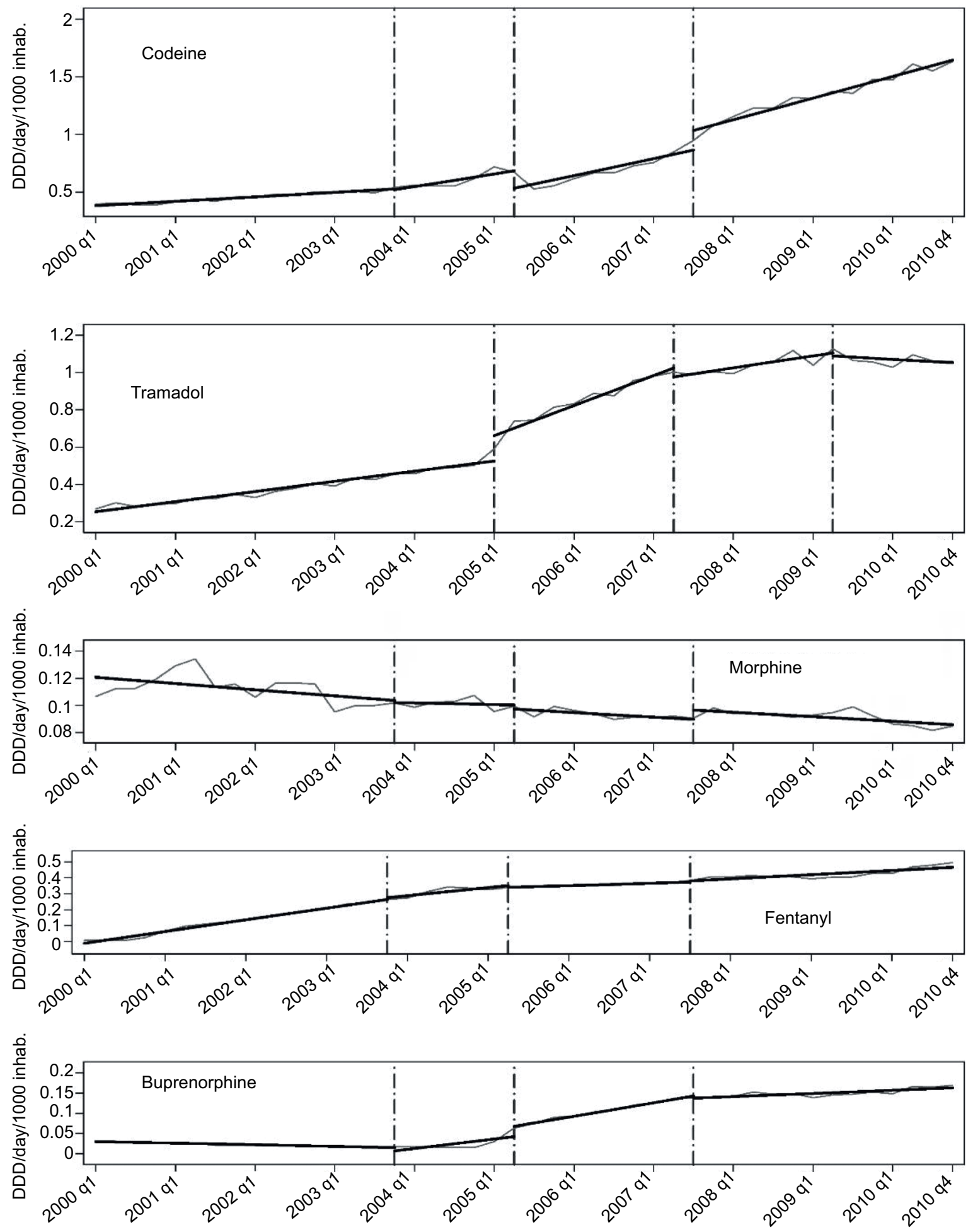

Figure 2 Segmented regression results for opioids sales to community pharmacies between 2000 and 20I0. Data in DDDs/day/I000 inhabitants (note the different scales). Dashed lines indicate estimated breakpoints.

Abbreviations: DDD, defined daily dose; inhab, inhabitants; q, quarter.

to the influence of regulation and marketing on prescribing physicians.

The analysis shows that sales of all the five opioids were affected by at least one relevant non-clinical factor between
2000 and 2010 (Figure 3). Furthermore, as previously reported by Mercadante, ${ }^{5}$ the overall positive trend varies from drug to drug, suggesting that the considered factors affect the prescription of different opioids to different degrees. 


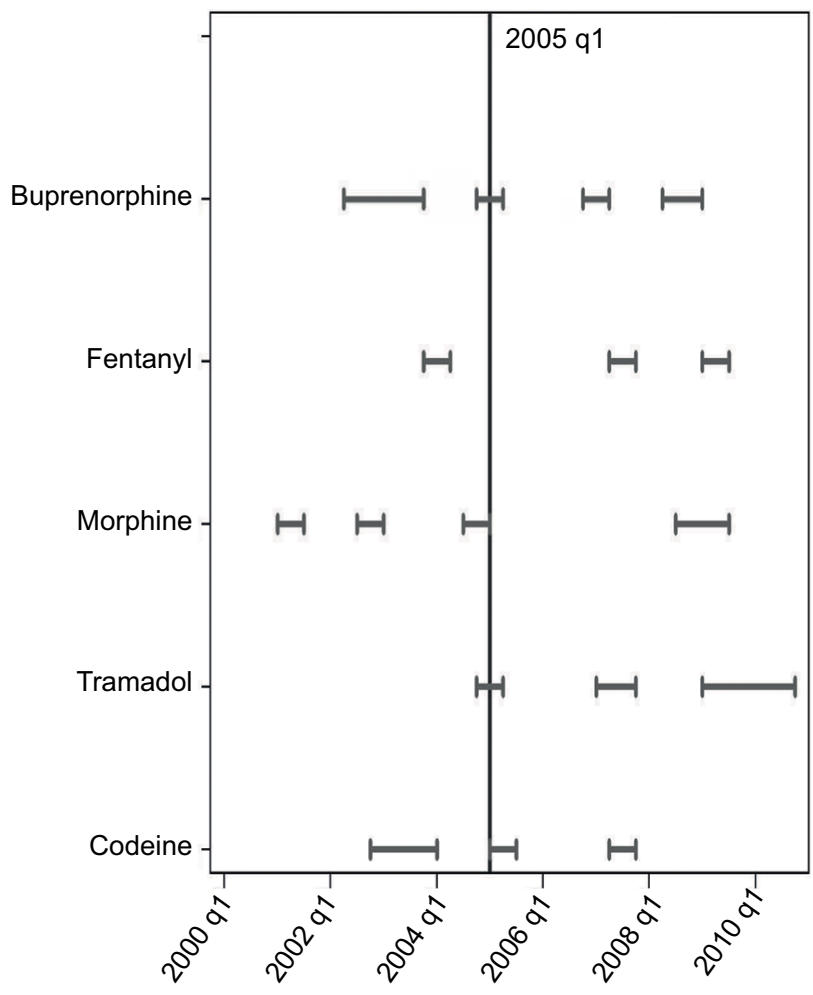

Figure 3 Overall synthesis of breakpoints estimated using segmented linear regression. The segments indicate the $95 \% \mathrm{Cls}$ of the break point estimates, for each drug considered.

Abbreviation: Cls, confidence intervals; q, quarter.

\section{The effect of technological innovation}

The influence of technological innovation on opioid consumption may be better analyzed comparing the trends of step III opioids. Fentanyl and morphine sales (Figure 2) were comparable to that of other European Countries, ${ }^{22}$ and almost superimposable to data observed in Nordic Countries ${ }^{7}$ and Israel. ${ }^{23}$ The sales of fentanyl increased from 0.011 to 0.468 DDDs/day/1000 inhabitants between 2000 and 2010, whereas morphine sales decreased from 0.113 to 0.084 DDDs/day/1000 inhabitants in the same period (Figure 2).

The positive trend of fentanyl sales may be due to a synergy of regulatory, marketing, and technological factors. As reported by Chinellato et al, ${ }^{24}$ the regulatory interventions induced a higher growth rate in fentanyl rather than in oral morphine prescriptions, even though no difference in prescribing laws and reimbursement policies were in place (Figure 2). Indeed, oral morphine solution and fentanyl transdermal patches were added to the list of reimbursable drugs by the Italian NHS almost simultaneously. ${ }^{24}$ Therefore, the different response to regulation changes may be due to the technological differences between the two dosage forms: transdermal patches are considered non-invasive systemic delivery systems, easy to stop and more manageable in comparison to an oral syrup or tablet. Furthermore, their use is correlated to a lower risk of misuse and to higher patient compliance. ${ }^{25}$ Such advantages seem to affect physicians' willingness to prescribe transdermal fentanyl instead of oral morphine, despite recommendations in international guidelines to prescribe transdermal fentanyl only as an alternative to oral morphine. ${ }^{26,27}$

It is noteworthy to observe that the availability of a particular pharmaceutical form is more relevant to opioids sales by community pharmacies than by hospitals, where patients' compliance is less critical in assuring treatment persistence of patients. ${ }^{10}$ Physicians' concerns about the risk of misuse seems to be another critical issue in understanding prescription choices. Indeed, fentanyl showed increased sales after the technological switch from reservoir to matrix formulation in 2007 (M/T6; Table 1). This change was required, since pharmacovigilance alerts had highlighted the risk of abuse related to oral absorption of the fentanyl-containing gel present in the reservoir transdermal patches. ${ }^{28}$ The matrix transdermal patch minimizes the risk of recreational abuse (the less the ease of drug extraction from dosage form, the less the risk of abuse) and brings additional advantages, such as the possibility to cut the patch, allowing for a more manageable dosage.

The introduction of therapeutic alternatives to fentanyl, namely buprenorphine transdermal patches (M/T2; Table 1) and oxycodone (M/T3; Table 1), slightly affected sales in the first part of 2004 (Figure 2). As in the case of transdermal fentanyl, buprenorphine showed an increase in sales when transdermal patches were made available in the second Quarter of 2003 (M/T2; Table 1). However, the results of transdermal buprenorphine are far from that of transdermal fentanyl in terms of sales (Figure 1), suggesting that greater caution is used by physicians in prescribing transdermal buprenorphine. Physicians' preference for transdermal fentanyl was confirmed in 2005, when the increase in sales of transdermal buprenorphine, by then reimbursed by the NHS (Reim1; Table 1), was stopped by the availability of generic transdermal fentanyl on the market (M/T5; Table 1).

\section{The effect of reimbursement changes}

The effect of reimbursement changes on opioid sales may be better clarified by comparing the data of Step II opioids (codeine and tramadol). Sales were positively influenced by regulatory implementations (Figure 2), but differences were 
observed depending on reimbursement policies. In the last part of 2004, both drugs reported a growth in sales, when NHS reimbursement for patients suffering for severe pain due to neoplastic or degenerative disease was introduced. For codeine, there was a temporary trend inversion at $\mathrm{M}$ / T3 (Table 1). In the same period, tramadol sales showed an increase of comparable magnitude. Nevertheless, codeine consumption continued to increase after that, as the reimbursement restriction was removed (Reim1; Table 1) and prescription to all patients was made possible in 2007 (R2; Table 1). Tramadol prescriptions took advantage of a less stringent regulation, besides an improvement in reimbursement policies (Reim1; Table 1). However, a significant decrease in tramadol sales occurred at R2 and R4 (which mainly influence drug products included in the Annex III bis of Law n. 12 of 2001). Tramadol sales reached a plateau in the first part of 2007 (Figure 2), when the prescription of opioids listed in Annex III bis was extended to all patients (R2; Table 1).

\section{Conclusion}

Regulatory, technological, and reimbursement changes that occurred in Italy between 2000 and 2010 have significantly influenced opioid sales to community pharmacies. Among the factors taken into consideration, reimbursement changes seem to have had a strong effect on sales, confirming the predominant role of the Italian NHS as a service provider in the assurance of public healthcare. However, different drugs where affected to different degrees, suggesting that sales may also be influenced by other elements, namely, marketing policies or the application of superseded clinical guidelines and protocols.

All the factors mentioned above may affect physicians' awareness about a specific medicinal product, influencing their prescribing choices. The introduction of oxycodone slow release preparations in 2004 is concurrent with a decrease in the use of morphine, despite the lack of any evidence of clinical differences or significant changes in the administration route or compliance. ${ }^{26}$ The increase in published articles in peer-reviewed health sciences journals between 2000 and 2010 was $\sim 3$-times higher for oxycodone $(+504 \%)$ than morphine $(+179 \%) .{ }^{29}$ The higher interest for oxycodone by the scientific community is a consequence of its central role in the opioid crisis. ${ }^{8}$

In conclusion, the higher the physicians' awareness about a drug, the higher the impact of regulatory, technological, and reimbursement changes on their prescribing practice. The sales of relatively new drugs (e.g., buprenorphine) seem to be less affected by changes in reimbursement and regulatory policies than that of established products (eg, codeine or fentanyl). Indeed, physicians are more comfortable with "old" drugs, since their clinical use is supported by established clinical guidelines and protocols. On the contrary, new drugs may lack such information, inducing physicians to prescribe them cautiously to avoid prescribing errors.

\section{Acknowledgments}

Data source: IMS Health S.r.l. Italian Retail Market years 2000-2010. The authors wish to thank IMS Health S.r.l. (now IQVIA) for kindly providing opioid sales data and Dr Giulia Torresin, for her contribution to data analysis.

\section{Disclosure}

$\mathrm{CB}$ has received consultancy fees from Molteni Farmaceutici and from Mundipharma Pharmaceuticals. AC has received consultancy fees from Italfarmaco, Prostrakan, Mundipharma Pharmaceuticals, Amgen, Grunenthal, Molteni farmaceutici, Novartis. CB has received a training grant from the Floriani Foundation - Milan (del.CDA 22/11/12). The authors report no other conflicts of interest in this work.

\section{References}

1. Schottenfeld D, Beebe-Dimmer JL, Buffler PA, Omenn GS. Current perspective on the global and United States cancer burden attributable to lifestyle and environmental risk factors. Annu Rev Public Health. 2013;34:97-117.

2. World Health Organization (WHO). Cancer Pain Relief: With A Guide to Opioid Availability, 2nd Edition. Geneva: WHO; 1996.

3. Kumar N. Report of a Delphi Study to determine the need for guidelines and to identify the number and topics of guidelines that should be developed by WHO. Geneva: WHO; 2007.

4. Cherny NI, Baselga J, de Conno F, Radbruch L. Formulary availability and regulatory barriers to accessibility of opioids for cancer pain in Europe: a report from the ESMO/EAPC Opioid Policy Initiative. Ann Oncol. 2010;21(3):615-626.

5. Mercadante S. Opioid prescription in Italy: new law, no effect. Lancet. 2002;360(9341):1254-1255.

6. Chinellato A, Skaper SD, Giusti P, Debetto P. Consumption of opioid analgesics in Italy: light at the end of the tunnel? Eur J Pain. 2011;15(2):220-221.

7. Hamunen K, Paakkari P, Kalso E. Trends in opioid consumption in the Nordic countries 2002-2006. Eur J Pain. 2009;13(9):954-962.

8. Grosser T, Woolf CJ, FitzGerald GA. Time for nonaddictive relief of pain. Science. 2017;355(6329):1026-1027.

9. Volkow ND, Collins FS. The role of science in addressing the opioid crisis. N Engl J Med. 2017;377(4):391-394.

10. Caraceni AT, Brunelli C, Rocco P, Minghetti P. Trends in opioid analgesics sales to community pharmacies and hospitals in Italy (2000-2010). Minerva Anestesiol. 2013;79(8):906-914.

11. Osservatoriosull'impiego dei medicinali (OsMed). National Report on Medicines use in Italy. Year 2015 (Italian edition), p 478. Available from: http://www.aifa.gov.it/sites/default/files/Rapporto_OsMed_2015_ AIFA.pdf.

12. Various decrees of the Italian Medicines Agency (AIFA), published in G.U. n.1, January 3, 2005. RomaIstituto Poligrafico eZecca dello Stato, 2005. 
13. Italian Medicines Agency (AIFA). In: Istituto Poligrafico e Zecca dello Stato; Decision of March 9, 2005 (G.U. n. 65 of 19 March 2005); Roma: AIFA; 2005.

14. Italian Medicines Agency (AIFA). In: Istituto Poligrafico e Zecca dello stato; Decision of September 22, 2009 (G.U. n. 238 del 13.10.2009). Roma: AIFA; 2009

15. World Health Organization (WHO). WHO Collaborating Centre for Drug Statistics Methodology, 2015. Available from: http://www.whocc. no/atc_ddd_index/?code=N02A. Last accessed January 22, 2018.

16. ISTAT: Portale per gli operatori delle statistiche demografiche. ISTAT, 2015. Available from: http://demografiche.istat.it/. Last accessed January 22, 2018.

17. Bai J, Perron P. Estimating and testing linear models with multiple structural changes. Econometrica. 1998;66(1):47-78.

18. StataCorp. Stata Statistical Software: Release 13. College Station, TX: StataCorp LP; 2013.

19. R Core Team. R: A language and environment for statistical computing. R Foundation for Statistical Computing. Version 3.0.1. Vienna: R Foundation for Statistical Computing; 2013.

20. Zeileis A, Leisch F, Hornik K, Kleiber C. strucchange: an R package for testing for structural change in linear regression models. J Stat Softw. 2002;7:1-38.

21. Ruscitto A, Smith BH, Guthrie B. Changes in opioid and other analgesic use 1995-2010: repeated cross-sectional analysis of dispensed prescribing for a large geographical population in Scotland. Eur J Pain. 2015;19(1):59-66.
22. Pain \& Policy Studies Group, University of Wisconsin/WHO Collaborating Center. Euro opioid consumption (2015). Available from: http://www.painpolicy.wisc.edu/who-regional-office-europe-euro. Last accessed January 22, 2018.

23. Ponizovsky AM, Marom E, Zeldin A, Cherny NI. Trends in opioid analgesics consumption, Israel, 2000-2008. Eur J Clin Pharmacol. 2011;67(2):165-168.

24. Chinellato A, Terrazzani G, Walley T, Giusti P. Opioids in Italy: is marketing more powerful than the law? Lancet. 2003;362(9377):78.

25. Gourlay GK. Treatment of cancer pain with transdermal fentanyl. Lancet Oncol. 2001;2(3):165-172.

26. Hanks GW, Conno F, Cherny N, et al. Morphine and alternative opioids in cancer pain: the EAPC recommendations. $\mathrm{Br} J$ Cancer. 2001;84(5):587-593.

27. Caraceni A, Hanks G, Kaasa S, et al. Use of opioid analgesics in the treatment of cancer pain: evidence-based recommendations from the EAPC. Lancet Oncol. 2012;13(2):e58-e68.

28. Sellers EM, Schuller R, Romach MK, Horbay GL. Relative abuse potential of opioid formulations in Canada: a structured field study. $J$ Opioid Manag. 2006;2(4):219-227.

29. Elsevier Scopus. "Analyze search results" tool; Reseach Keys: "Opioid Name” AND "Pain”, SUBJAREA (MEDI OR NURS OR VETE OR DENT OR HEAL OR MULT). Available at: https://www.scopus.com/ search/form.uri?display=authorLookup. Accessed June 14, 2016.
Journal of Pain Research

\section{Publish your work in this journal}

The Journal of Pain Research is an international, peer reviewed, open access, online journal that welcomes laboratory and clinical findings in the fields of pain research and the prevention and management of pain. Original research, reviews, symposium reports, hypothesis formation and commentaries are all considered for publication.

\section{Dovepress}

The manuscript management system is completely online and includes a very quick and fair peer-review system, which is all easy to use. Visit http://www.dovepress.com/testimonials.php to read real quotes from published authors. 